\title{
Interrogation of Elegant System for Regulating Vanet Traffic with Probable Path Planning
}

\section{Rajiv GG*}

Department of ECE, VSB College of Engineering Technical Campus, Coimbatore, India

\begin{abstract}
Technology is grooming in all discipline lead to the development of automation in various domains. Incorporating automation in various devices will make the device to work smart. Though this automation does not conquer the automobile industry fully, it made vehicles as semi-automated by employing route navigation facility methods to avoid front end and rear end collision, etc. The evolution of automation in the automotive domain has paved the way for the improvisation in that sector. Though the existing methodology has proved to be working smoothly, it encounters few limitations in turns of accuracy, traffic/congestion monitoring etc. The proposed Smart Traffic support System (STS) improves the accuracy of the above mentioned parameters by integrating the advantages of both ad-hoc network and the cellular communication. The inclusion of real time path planning algorithm will provide an enhanced traffic management by suggesting various alternative paths instead of one.
\end{abstract}

Keywords: Vehicle ad hoc networks (VANET), Road side unit (RSU), Video on demad service (VoD)

\section{Introduction}

The recent survey by Google states that one billion users are using Google maps in a day across the globe. This lead to further more exploring the promising technology of autonomous car navigation system supported with GPS. Era of wireless technology equipped with sensor nodes made it possible to define the routes for any location; this technology can be cumulatively referred to as VANET which is a combination of sensor nodes with ad hoc networks. VANET takes ultimate challenge of receiving information from nodes which is deployed on roadside sensor which senses data of the vehicles moving in a random fashion [1]. At such condition measuring the accuracy of traffic will remain to be a challenging task. Automation in auto mobile industry emphasis on better navigation system, so demand in this technique is raised gradually that incorporates up gradation directly in car navigation system and indirectly in coverage limit of VANET.

\section{Early Studies}

Automobiles which are manufactured in recent years are supported with navigation service system. Navigation Services which is available in vehicle provides high resolution traffic information to drivers. But traffic congestion information can be effectively analyzed for only monitored roadways, Scenario is totally different for unmonitored roadways. Hence unmonitored roadways needs keen attention in data collection and a better traffic estimation algorithm is needed to process the data. In conventional traffic monitoring method the data collection is accomplished by sensor networks [2]. Main Challenge in sensor network is deployment of such sensors in large area becomes expensive and maintenance of sensing system is a tedious job. As a result thereby monitoring roads during prime time and rush hours is difficult.

This drawback can be solved [3] by improving the coverage and timeliness through GPS probe vehicle based system. This method could potentially improve the collection of real time traffic condition over complete network with minimal implantation cost. The reliability of such system is validated through analysis, simulation and experiments. Real time implementation of system is achieved by triggering up the privacy mechanism scheme where the prerequisite is most trusted centralized privacy servers. The development of IEEE $802.11 \mathrm{p}$ with extended features of protocols which is guided with mobile multimedia services that claims higher bandwidth services [4] in various road scenarios for driver and passenger. This led to the new inventions on VANET. VANET aided with VoD (Video on Demand Service) has undergone two main challenges during implementation because of lack of interaction to improve user quality level.

First main Challenge is the random behavior of user when interacting with the contents leads to data fragmentation, a primary cause for low sharing efficiency and high maintenance cost. Second main challenge is due to mobility of nodes the geographical distance between nodes changes rapidly. Hence, a better hybrid solution is needed to be framed for such VANET supported heterogeneous network.

In another method the grouping of community [5] is based on playback and movement of nodes of similar behavior. This is carried out by Fuzzy ant clustering algorithm and mobility similarity measurement model. Ant colony clustering and fuzzy $\mathrm{C}$ means is used to find nodes of similar playback and Markov process is used to locate the mobility of nodes. The maintenance process is done by Mobile Community Management Mechanism (MCMM) which takes care of role and task of users in the community, cumulative storage. Travel Time Index (TTI) is a ratio of travel time in rush hours to ratio of travel time in quiet period. TTI says, due to phenomenal increase in traffic congestion enormous time is wasted on road during a travel. To overcome this drawback many smart and intelligent traffic management system is been implemented and such system does not guarantee successful congestion control at traffic as it claims [5,6].

*Corresponding author: Rajiv Gandhi, Assistant Professor, Department of ECE, VSB College of Engineering Technical campus, Coimbatore, India, Tel: 8220048212; E-mail: rajeevgurusamy@gmail.com

Received November 20, 2018; Accepted November 22, 2018; Published November 30, 2018

Citation: Rajiv GG (2018) Interrogation of Elegant System for Regulating Vanet Traffic with Probable Path Planning. J Telecommun Syst Manage 7: 174. doi: 10.4172/2167-0919.1000174

Copyright: (c) 2018 Rajiv GG. This is an open-access article distributed under the terms of the Creative Commons Attribution License, which permits unrestricted use, distribution, and reproduction in any medium, provided the original author and source are credited. 
Many researches has proved that VANET can anticipate congestion on road and it can ensures the road safety by altering the drivers regarding the traffic information and collision with front end or rear end vehicle there by avoiding collisions on road [6,7]. Next level of VANET supports query mechanism, that means if a driver is not aware of road and needs to location of toll station or petrol bunk, he/she can raise a query about it while it takes one to ten miles to reach the broadcast site finally information will reach drivers within the acceptable range of minimal delay. As emphasized [8] the method provides sufficient data delivery with reasonable delay whenever a query is raised by driver in any kind of network architecture.

If network is scarcely distributed Vehicle Assisted Data Delivery (VADD) method routes the query packet in another alternative path and receives reply at tolerant delay time. VADD uses carry and forward technique, the query is carried to broadcast station and if any issues at path then the packet is forwarded to another path within the coverage of network.

VANET is well developed and groomed technology as far as wireless sensor nodes is concerned. Deployment of wireless gateways on road side unSTS (RSU) developed another dimension on smart traffic management by grooming vehicles with on- board communication, vehicle to RSU communication and vehicle to vehicle communication [7]. Asymptotic laws are based on concept of probability of a vehicle can use multiple path within the particular capacity. Asymptotic throughput can bound number of RSUs to be deployed in a network.

As mentioned [9] challenges are addressed by i) evaluating the asymptotic throughput capacity during the uplink scenario ii) scalability of RSU is determined iii) to improve throughput performance.

This method is further improved by using intelligent packet forwarding to server with optimized throughput performance. The proposed STS overcomes the above short comings by combining VANET with cellular communication [10] which in turn will provide an efficient vehicle to vehicle communication as well as monitoring.

\section{Proposed Smart Transport Support System}

Vehicle to Vehicle (V2V) communication is considered to be one of the most tedious process because of the difficulty in capturing and monitoring the information obtained from the adjacent vehicles, road side unSTS etc. The proposed STS incorporate both Vehicle Ad-Hoc network and cellular communication for the enhanced traffic control and monitoring. In this method the information obtained are shared among the vehicles [11] Road Side UnSTS (RSU) and traffic server. The traffic server plays the key role in identifying the alternative path and forwarding the location of collision to the nearby hospital or police station in case of any emergency. In addition the proposed method applies real-time path planning algorithm to get as much of alternative shortest paths during congestion instead of a single alternative path. In Hybrid-VANET based transportation system, [12] shown in Figure 1, Vehicles are equipped with the on-board unSTS that enable Multihop $\mathrm{V} 2 \mathrm{~V}$ communication which inturn is used to deliver the periodic vehicle information (e.g., vehicle velocity, density, and location). This information is collected using sensor unSTS or camera. When vehicles sense accident-related information like congestion, a warning message is generated to alert the emergent accident information and then is shared not only among vehicles but with the nearest RSU via Vehicle to Road Side Unit (V2R) communications. Hence, the taxis or buses can directly upload the received warning message to the nearest cellular BS, and the BS will deliver the message to the vehicle traffic server.

RSUs deployed along the roads obtain vehicle-traffic statistical information (i.e, the vehicle arrival/ departure rate on each road). Here the taxis and buses are perfectly connected to the cellular system, and RSUs are well connected with each other through wire line.

If RSUs are deployed at intersections, the traffic information can be detected by the equipped cameras or traffic flow meters connected to RSUs directly. Generally, vehicular networks contain two types of



Figure 1: Intelligent traffic system 
nodes: vehicles and roadside stations. Both possess Dedicated ShortRange Communications (DSRC) devices. DSRC works in $5.9 \mathrm{GHz}$ band with bandwidth of $75 \mathrm{MHz}$ and approximate range of $1000 \mathrm{~m}$ [13] The network should support both private data communications and public (mainly safety) communications but higher priority is given to public communications.

Here in STS, Vehicle will work as a node and build the network to get up to date information about traffic condition on the road on different available paths to reach the destination from the source. This will make it easy for the owner to take decision of selecting path based on available alternative path considering time and distance parameters. As vehicle progresses on road, it will get information about the current status of traffic. When vehicle head towards destination, if there is traffic on the route it is following and if alternate path is available, base station will send message about the available alternate path to leading vehicle on that route and traffic server plan many alternate path using time based path planning algorithm and it is displayed in the digital display on the road side unit [14]. Digital display helps in case of any failure in the message transmission. Due to many alternate path vehicles can move through different alternate paths.

The traffic server will perform the real time path planning algorithm based on the obtained information and will in-turn provides various alternative paths. The information about the paths will be shared with the vehicles, RSU and will also be displayed in the monitors positioned on the roads as shown in Figure 2. The digital monitors will forecast the information like the congestion occurrence, alternative paths based on distance, climate condition etc., which will serve as a backup for the user in case of any hindrance in delivering the message to the user directly [15].

The user can chose the path based on their preference. Also road side facilities like petrol pump, restaurants, entertainment centers, shopping malls, hospitals, banks, schools etc. information is mapped in the system and same can be communicated to the user on the navigation map. This system makes user more informed about the on route available facilities. User can take the required route if he wants to use the facility. This way this system can act as user friendly smart system which will guide driver as he proceeds on road.

If an accident is detected by the traffic server it will convey the information about the accident's location to the nearby hospitals and police station using cellular communication. The proposed method uses kruskal's algorithm to determine the shortest path among the available alternative paths.

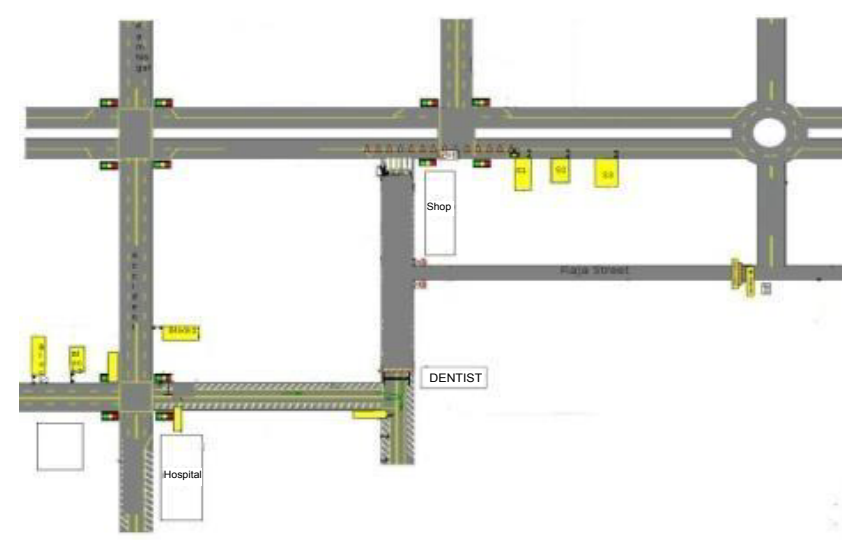

Figure 2: Path Planning in STS.
The dynamic source routing is used to communicate the information due to STS efficient routing. The dynamic source routing is demand based routing technique in which the route is established only when it is required. Unlike table driven approach the need to find routes to all other nodes in the network is eliminated. The intermediate nodes also utilize the route cache information efficiently to reduce the control overhead and enhance the bandwidth.

\section{Result and Discussion}

To collect time-varying traffic-condition information, most works in conventional STS usually rely on cellular systems or loop detectors. Exhaustive Collect ion of real-time traffic information for traffic forecast or reconstruction in experimental research is done which is shortly described in Table 1.

In a traffic management system with loop detectors for continuous traffic measurement and monitoring along arterials is introduced. However, inevitable drawbacks cast a shadow on the application of cellular systems and loop detectors. For cellular systems, as they are not dedicated for traffic data collection, the collection services can be highly costly, and the high volume of traffic data may also cause congestion for other cellular services. For the loop detectors, the deployment expense can also be very high. The improper distance measurement leads to incorrect path planning. Due to VANETs, V2V and V2R communications can make real-time message delivery much quicker, cheaper, and more efficient than the current systems, even for short-distance transmissions in dense networks. Hence, in this method to reduce the end-to-end transmission delay, taxis or buses are considered as super relays to help in delivering the information through the cellular network of public transportation system.

Due to VANETs, V2V and V2R communications can make realtime message delivery much quicker, cheaper, and more efficient than the current systems, even for short-distance transmissions in dense networks. Hence, in this method to reduce the end-to-end transmission delay, taxis or buses are considered as super relays to help in delivering the information through the cellular network of public transportation system.

The Figure 3 shows the variation in time taken to reach the

\begin{tabular}{|l|c|}
\hline Simulation area & $1500 \mathrm{~m} \times 1500 \mathrm{~m}$ \\
\hline Vehicles Cluster Size/lane & $150-200$ \\
\hline Transmission range & $300 \mathrm{~m}$ \\
\hline Simulation time & $500 \mathrm{~s}$ \\
\hline Vehicle velocity & $50-60 \mathrm{kmh}$ \\
\hline
\end{tabular}

Table 1: Traffic information.

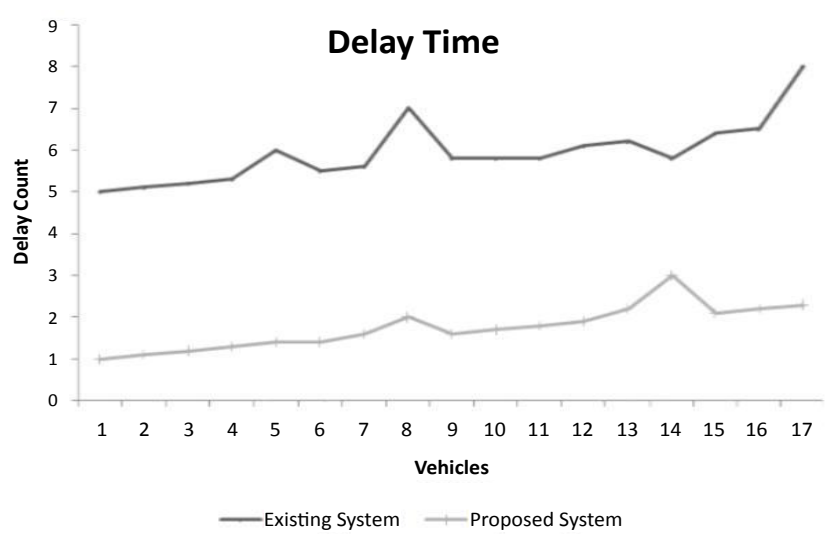

Figure 3: Destination reaching time. 
destination. A study is done to compare the traditional method and STS. It is evident that the designed STS functions better and the time taken to reach the destination is reduced comparatively Figure 4 shows the traffic rate of the proposed method. Traffic flow theory refers to the traffic stream variables of speed, flow, and concentration. These relationships are mainly concerned with uninterrupted traffic flow, primarily found on freeways or expressways. In the proposed algorithm, even when the density of the vehicles increases, the congestion is prevented comparatively.

Figure 5, illustrate the network overhead analysis. Network overhead refers to metadata and network routing information sent by an application, which uses a portion of the available bandwidth of a communications protocol and is a reduced overhead, is observed in STS.

Figure 6 shows the number of vehicles cleared in case of congested road traffic. Though the road is misguided or blocked, the vehicles are directed to their destination path properly in STS.

It's evident from the scenario that the STS functions better and the number of vehicles subjected to smooth driving is increased.

\section{Conclusion}

Recent advances in wireless technologies have given rise to emergence of Vehicular Ad-hoc Networks (VANETs), which is used to provide traffic management, route planning, and identifying roadside amenities using short-range wireless communication. The proposed technique discuss about the efficient and effectiveness in managing the traffic by incorporating both VANET and cellular communication. Here a real time path planning algorithm is used which provide several alternative paths instead of a single alternative path like the existing



Figure 4: Traffic rate.

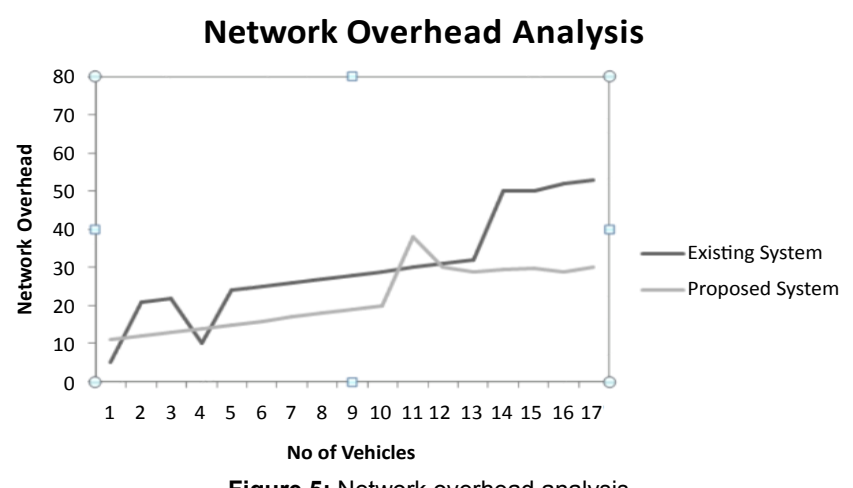

Figure 5: Network overhead analysis.

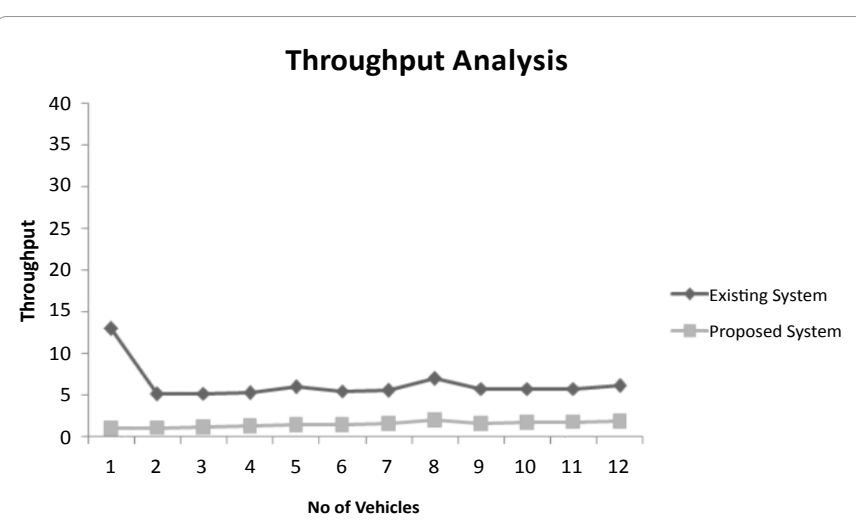

Figure 6: Throughput analysis.

methods. Here the shortest path among these alternate paths is find out which reduces the delay of vehicles to a more extent. And in RSU speed sensors and cumulative sensor are used for detecting the speed of the vehicles and analyzing the road condition. The usage of dynamic source routing improves the bandwidth by avoiding the periodic table update like table driven approach. This system also creates an immediate path during any collisions. The time taken to find an alternate route is minimized comparatively. And because of this, it can also be claimed that the carbon emission is reduced to a greater extent which results in saving of non-renewable energy source such as diesel/ petrol.

\section{References}

1. Seyed AS, Abdul HA, Wan HH, Mohammad HS, Shidrokh G, et al. (2015) Trust management in vehicular ad hoc network: a systematic review. EURASIP Journal on Wireless Communication and Networking

2. EL Amine AM, Drias H (2012) A Cooperative Multi-Agent System for Traffic Congestion Management in VANET. In: Wyld D, Zizka J, Nagamalai D (eds.) Advances in Computer Science, Engineering \& Applications. Advances in Intelligent and Soft Computing, Vol 166. Springer, Berlin, Heidelberg.

3. Baik H, Toch I, Quinn J, Daniel W, Alexandre MB, et al. (2012) Enhancing Privacy And Accuracy In Probe Vehicle-Based Traffic Monitoring Via Virtual Trip Lines. IEEE Trans Mob Computing 11: 1536-1233.

4. Xu C, Futao Z, Jianfeng G, Zhang H, Muntean GM (2013) QoE-driven usercentric $\mathrm{VoD}$ services in urbanmultihomed P2P-based vehicular networks. IEEE Trans Veh Technol 62: 2273-2289.

5. Ilias L, Gustavo, David Mack, Giovanni Pau, Cecilia Mascolo et al. (2011) On the effectiveness of an opportunistic traffic management system for vehicular networks. IEEE Trans Intell Transp Syst 12: 1537-1548.

6. Rak J (2013) Providing Differentiated Levels of Service Availability in VANET Communications. IEEE Communications Letters 111-145.

7. Jacek R (2014) LLA: A New Anypath Routing Scheme Providing Long Pat Lifetime in VANETS. IEEE Communications Letters 18: 167-256.

8. Jiming C, Weiqiang Xu, Shibo He, Youxian S, Preetha T, et al. (2010) UtilityBased Asynchronous Flow Control Algorithm for Wireless Sensor Networks. IEEE Journal On Selected Areas In Communications 28: 456-500.

9. Elias SJ, Mohd Warip MN, Mansor S, Muhamat Dawam SR, Mansor AR (2017) Experimental Model of Congestion Control Vehicular Ad Hoc Network Using OMNET++. In: Ahmad AR., Kor L., Ahmad I., Idrus Z. (eds) Proceedings of the International Conference on Computing, Mathematics and Statistics (iCMS 2015). Springer, Singapore

10. Jing Z, Cao G (2010) VADD: Vehicleassisted data delivery in vehicular ad hoc networks. Proc IEEE INFOCOM, Barcelona, Spain, pp: 1-12.

11. Miao W, Hangguan S, Tom HL, Ning Lu, Ran Z, et al. (2008) Asymptotic Throughput Capacity Analysis Of Vanets Exploiting Mobility Diversity. IEEE Trans Veh Technol 64: 4187-4202.

12. Drawil N, Basir O (2010) Inter vehicle communication-assisted localization IEEE Trans Intell Transp Syst 11: 678-691. 
Citation: Rajiv GG (2018) Interrogation of Elegant System for Regulating Vanet Traffic with Probable Path Planning. J Telecommun Syst Manage 7: 174. doi: $10.4172 / 2167-0919.1000174$

Page 5 of 5

13. Schoepflin T, Dailey D (2003) Dynamic camera calibration of roadside traffic management cameras for vehicle speed estimation. IEEE Trans Intell Transp Syst 4: 90-98.

14. Younshik C, Wilfred WR (2012) Methodological Approach For Estimating
Temporal and Spatial Extent Of Delays Caused By Freeway Accidents. IEEE Trans Intell Transp Syst 13: 1454-1461.

15. MiaoWang HS, Rongxing Lu, Ran Z, Xuemin S, Fan B (2015) Real-Time Path Planning Based on Hybrid-VANETEnhanced Transportation System. IEEE Trans Veh Technol 64: 1664-1678. 